\title{
Self-Serving Cheap Talk: A Test Of Aumann's Conjecture ${ }^{1}$
}

\author{
Gary Charness ${ }^{2}$ \\ Department of Economics and Business, Universitat Pompeu Fabra, \\ Barcelona, Spain 08005 and Department of Economics, Uinversity of California at \\ Berkeley, Berkeley, California 94903
}

Received September 30, 1998

\begin{abstract}
The effectiveness of pre-play communication in achieving efficient outcomes has long been a subject of controversy. Aumann conjectures that, in a variant of the Stag Hunt game, "an agreement to play [the efficient outcome] conveys no information about what the players will do" and thus should not affect behavior. Farrell and Rabin suspect that cheap talk will achieve efficiency. Results show impressive coordination when the sender first chooses a signal and then an action. Without communication, efforts to achieve efficiency are largely unsuccessful. As Farrell suggests, reversing the order of the action and signal appears to change players' behavior. Journal of Economic Literature Classification Numbers: A13, C72, C91, D82, D84. (๑) 2000 Academic Press
\end{abstract}

\section{INTRODUCTION}

While most economists would agree that communication can affect outcomes, the effectiveness of "cheap talk" (costless, non-binding pre-play communication) in achieving efficient outcomes is a subject of controversy. Since many economic interactions feature non-binding messages, this issue has considerable importance for efficiency and social welfare. ${ }^{3}$ Cheap talk should be effective in achieving coordination in some types of games, but not in others where interests conflict completely or where a signal pooling problem critically impairs the credibility of a message.

${ }^{1}$ I thank Jordi Brandts, Antonio Cabrales, Rosemarie Nagel, and Matthew Rabin for valuable suggestions and ideas. All errors are my own. The financial support of Spain's Ministry of Education under grant D101-7715 is gratefully acknowledged.

22E-mail: charness@upf.es and charness@econ.berkeley.edu.

${ }^{3}$ Farrell and Rabin (1996) argue that these situations are more common than those where costly signaling is relevant. 
Coordination games feature multiple equilibria. Often there is an efficient outcome which can only be achieved through coordination; some type of communication is possible, but there is no binding enforcement mechanism available. Sometimes we can rank the Nash equilibria on the basis of Pareto efficiency or payoff dominance, so that we can determine the efficient equilibrium and observe the frequency of its realization. Coordination failures are theoretically possible, particularly when there is a risk-dominant equilibrium (Harsanyi and Selten, 1988) which differs from the Pareto-efficient one.

Aumann (1990) expresses the view that communication cannot affect the outcome of the game if the signaler(s) has/have a strict preference over the other player's strategy choice. He illustrates this view with the following game

$\begin{array}{ccc} & \text { A } & \text { B } \\ \text { A } & 7,7 & 8,0 \\ \text { B } & 0,8 & 9,9 .\end{array}$

Both $[A, A]$ and $[B, B]$ are pure-strategy Nash equilibria, with $[B, B]$ payoffdominant and $[\mathrm{A}, \mathrm{A}]$ risk-dominant. Aumann points out that it in a player's interest to always signal B, regardless of whatever strategy she actually intends to use, since each strictly prefers that the other play B. Let us call such a game an Aumann game. He concludes (p. 206): "Therefore an agreement to play $\cdots[\mathrm{B}, \mathrm{B}] \cdots$ conveys no information about what the players will do, and cannot be considered self-enforcing."

The logic is impeccable, but some feel that an expectation that communication will not affect results is unrealistic. Farrell and Rabin (1996) state (p. 114): "although we see the force of Aumann's argument, we suspect that cheap talk will ... [achieve B,B]." Certainly it is true that the sender wants the receiver to believe the message whether or not she intends to act in accordance with the message-this is Aumann's point. However, one might also ask if the sender would wish to send an accurate message if she thought the receiver would believe the message. It all depends on beliefs and credibility; both views are internally consistent. ${ }^{4}$

Farrell (1988) also writes (p. 213) that "it is a matter of whether [a player] decides on his move ... after he chooses his message, or ... his move first and then his message. If the latter, then Aumann's criticism is compelling; if the former, then matters are rather unclear." The intuition is that the participants' perceptions of the salience of the self-interest issue may well

${ }^{4}$ I thank Matthew Rabin for useful comments in this regard. 
influence the credibility of the potentially misleading and self-serving message.

Previous experimental work suggests that the extent to which communication can enhance coordination may well vary across different forms of games and message technologies, as results with pre-play communication have varied. This paper reports the results of a test of Aumann's conjecture, using one-way written signals marked on message forms. When the signal is followed by an action, there is a high degree of coordination on the payoff-dominant equilibrium. This percentage does not appear to be very sensitive to the degree of risk dominance in the game. When the game is played without communication, risk dominance generally prevails, despite some attempts to achieve the payoff-dominant outcome. In a prisoners' dilemma control treatment, the cooperative move is generally signaled, but rarely played by either sender or receiver.

Since the results in the control sessions are so different from those when the signal precedes the action choice, it seems clear that a B signal does convey information. One cannot conclude that simply because a message could be self-serving, it will be ignored. The behavioral question (on which a payoff-maximizing player should base a choice of actions) is what actions people actually take, given a particular signal. The purely logical argument that potential self-servingness necessarily destroys credibility is clearly refuted by the data.

The Farrell (1988) comment is also tested, by (physically) requiring that the sender choose an action prior to sending a message. Here behavior is more consistent with Aumann's conjecture. This environment is highly unpredictable, but the results for this treatment and a control treatment without communication are not dramatically different. ${ }^{5}$ Farrell's point about behavior being sensitive to the order of announcements and actions does appear to matter empirically.

In Section 2 of this paper, I mention some theoretical issues pertaining to coordination and communication, as well as relevant experimental results. Section 3 describes the experimental design and the results are presented in Section 4. Section 5 offers some discussion of the results and issues affecting beliefs about conditional play. Section 6 concludes and presents extensions to "hierarchies" of cheap talk and potential classifications of types of games by the degree of conflict or coordination.

${ }^{5}$ From the standpoint of statistical significance, the degree to which the results differ depends on the restrictiveness of the test used. See Section 4. 


\section{COORDINATION, COMMUNICATION, AND DOMINANCE}

When players' interests are well-aligned, communication can readily achieve coordination. As an example, consider the battle of the sexes

$\begin{array}{ccc} & \text { B } & \text { F } \\ \text { B } & 2,1 & 0,0 \\ \text { F } & 0,0 & 1,2 .\end{array}$

With a one-way signal, it should be easy to achieve $[\mathrm{B}, \mathrm{B}]$ or $[\mathrm{F}, \mathrm{F}]$; the outcome depends on which player is chosen to signal. In this game there is no reason to attempt to deceive the other player. The message is selfsignaling, as the signaler wishes to convey information if and only if it is true. However, note that coordination is much more problematic here with two-way simultaneous messages.

Farrell and Rabin (1996) define a message to be self-committing if it creates incentives for the signaler to fulfill it, if the other player considers the message credible. A credible message must be self-committing. Yet, selfcommitting messages need not be entirely credible or, as in Aumann (1990), self-enforcing. In the background, there is the issue of payoff-dominance versus risk-dominance. Harsanyi and Selten (1988) find both to be quite relevant in equilibrium selection. On the other hand, Harsanyi (1995) states (p. 94) that "further reflection on the Aumann (1990) arguments has convinced me ... [to] use only risk dominance as choice criterion among different equilibria without any use of payoff-dominance." So the issue of whether payoff-dominance is relevant still seems to be unsettled.

Blume (1998) finds that effective one-sided communication can be a stable outcome if the preferred equilibrium is not risk-dominated. ${ }^{6}$ Hurkens (1996) examines the question in games where messages are costly (moneyburning) and concludes (for one-sided pre-play communication) that the signaler must achieve his or her preferred outcome. ${ }^{7}$ The cost $(\varepsilon)$ can be arbitrarily small. If we consider a false signal to have even a small psychological disutility for the sender, this model predicts successful coordination on the payoff-dominant equilibrium.

${ }^{6}$ Blume notes that in this type of game $(2 \times 2$ Aumann game $)$, the payoff-dominant and risk-dominant equilibrium may be the same. An example is the game below:

$\begin{array}{ccc} & \text { A } & \text { B } \\ \text { A } & 7,7 & 8,6.9 \\ \text { B } & 6.9,8 & 9,9 .\end{array}$

${ }^{7}$ Both Blume and Hurkens use curb (closed under rational behavior) retracts as the cornerstone of the analysis. 
It is easy to calculate the minimum probability assessment required for a neo-classical agent to play $\mathrm{B}$ in response to a $\mathrm{B}$ signal. ${ }^{8}$ In a sense, it is this subjective probability estimate that reflects the degree of perceived risk. Even if we neglect any non-pecuniary "warm glow" from achieving efficiency, there is another issue: if the sender assigns a high probability that his signal will be considered to be credible, he does best by playing B. Is it reasonable to believe that the receiver considers the message sufficiently credible? Perhaps so; this is a behavioral question. The answer may be related to the extent of the influence of payoff-dominance in the population.

Evidence from experimental tests of coordination in games is rather mixed. Isaac and Walker (1988) find that communication is very effective in a public goods experiment. Cooper et al. (1990) observe that forward induction has limited success in generating coordination. Van Huyck et al. (1990) find convergence to the payoff dominant outcome with small fixed groups of subjects, but coordination failure with large groups.

Van Huyck et al. (1993) employ a two-stage design to test forward induction: the first stage consists of an auction for the right to play in a median-effort coordination game, where there are ranked equilibria and deviations from the median action are costly. There is a high degree of convergence toward the payoff-dominant equilibrium using the 1st-stage auction, while this convergence is never observed without an auction. ${ }^{9} \mathrm{Ca}-$ chon and Camerer (1996) also use the median game and transform the payoffs in later rounds, so that a positive net payoff is only possible with coordination on a high median value for the group. They observe movement toward higher-ranked equilibria when there is common knowledge of the payoff transformation, suggesting that loss-avoidance is a selection principle. $^{10}$

There are few tests of the effectiveness of pre-play communication with respect to coordination. One game (SCG) tested in Cooper et al. (1992) is

A

$$
\begin{array}{ccc}
\text { A } & 800,800 & 800,0 \\
\text { B } & 0,800 & 1000,1000 .
\end{array}
$$

Three conditions are tested: no signals, one-way signals, and simultaneous two-way signals. They report that (for the last 11 rounds of the 22 played)

\footnotetext{
${ }^{8}$ The critical probabilities are $2 / 3,5 / 6$, and $7 / 8$ for B play in the three calibrations tested. The mixed strategy equilibrium occurs when both players mix their plays with these (respective) probabilities.

${ }^{9}$ However, as Ochs (1995) points out, there may be a confound due to a selection bias, so the interpretation is not necessarily clear.

${ }^{10}$ The median action is unaffected if this payoff transformation is only private knowledge.
} 
the efficient outcome is reached in $0 \%, 53 \%$, and $90 \%$ of the cases, respectively. Although they conclude that only two-way communication is effective in achieving efficiency, a one-way signal also has a significant and substantial effect on coordination. However, their payoffs do not permit a direct test of the Aumann conjecture, as there is no strict preference over the other player's action when he or she intends to play A.

Clark et al. (1997) do examine the Aumann conjecture directly ${ }^{11}$

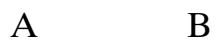
A $700,700 \quad 900,0$
B $\quad 0,900 \quad 1000,1000$.

Two-way signals are used and a no-communication treatment is also conducted. Clark et al. find that the likelihood that B is played is $42 \%$, even though $\mathrm{B}$ is signaled $81 \%$ of the time, in contrast with the Cooper et al. (1992) two-way signal results and their own replication of these SCG results. ${ }^{12}$ However, the corresponding percentage of $\mathrm{B}$ plays in the game without signals is $19 \%$ and they state (p. 11) that "the proportion of [B] choices $\ldots$ is affected by communication $(p=0.0027)$." Nonetheless, the efficient outcome is not observed in most cases and they conclude on p. 14 that "these results suggest that communication is not sufficient for attaining efficiency in simple coordination games."

In these tests, the risk-dominant equilibrium is generally observed when signals are not possible, while the payoff-dominant equilibrium is far more prevalent with signals. Even though the degree of effectiveness of communication varies, signals seem to always significantly increase the likelihood of efficient play in coordination games. But there has been only limited success for one-way signals in experimental $2 \times 2$ coordination games, even though there is some theoretical basis for expecting coordination.

\section{EXPERIMENTAL DESIGN}

Sessions were conducted at Universitat Pompeu Fabra in Barcelona. A total of 252 people participated, with each present at one session. Subjects were recruited by posting notices and were almost exclusively economics,

${ }^{11} \mathrm{I}$ became aware of this paper only after conducting my experimental sessions.

${ }^{12}$ Clark et al. find that, in the SCG, subjects played B in $77 \%$ of all cases, while the probability of a B signal was $85 \%$. Overall in their Aumann game, the proportion of B choices conditional on a $[\mathrm{B}, \mathrm{B}]$ announcement was only $50 \%$, compared to $96 \%$ in the replication of Cooper et al. 
business, and humanities undergraduate students. Average earnings were about $\$ 10.00$, including a show-up fee. The experimental instructions are available upon request.

There were generally 12 or 18 people in each session. ${ }^{13}$ Subjects were seated at carrels separated by dividers and a ban on verbal communication was enforced. Instructions (available upon request) were given to each person and were also read aloud to ensure a common knowledge condition. There were 10 periods in each session; participants were told that there would be random re-matching in each period and that the role of the signaler was also randomly drawn for each period. Random matching only took place within subgroups of six players. ${ }^{14}$ While it is true that two people were sometimes paired more than once, no player was ever aware of the identity of the other player in the pair. Since there were generally 18 participants in a session, subjects had little reason to be concerned with repeat game effects, as they did not know that pairings were only formed within these six-person groups.

Prior to each period, every participant was given a piece of paper. Senders received a paper which read "I intend to play [A or B]." 15 For receivers, the initial paper received was blank. Identical black pens were provided to each person, with which the signal was indicated on the paper. In this way, no one knew which people made signals in a round. After signals were marked, papers were collected and sorted; signals were distributed to assigned receivers and senders received blank pieces of paper. At this point, players marked play choices on a decision form. These forms were collected and the payoffs were determined and marked on the forms, which were then returned to the participants. Payoffs were aggregated over the 10 periods in the session and subjects were paid individually and privately.

Three different payoff calibrations were used in the coordination game, to investigate the degree of sensitivity to risk-dominance. ( 1 point $=$ 1 peseta, $\$ 1.00 \cong 150$ pesetas). These, along with the prisoner's dilemma played, are shown in Table I.

In the AS treatment, game 2 was played. Senders completed their decision sheets before receiving message forms; as in the other signal treat-

\footnotetext{
${ }^{13}$ Two sessions had only 6 subjects.

${ }^{14}$ The point of this design is to obtain independent data points, since each group's actions are internally interdependent. With separate groups of 6 , we can treat each cell as a single independent data point. I am indebted to Rosemarie Nagel for this suggestion.

${ }^{15}$ This signal was in Catalan, with translation by Andreu Mas-Colell. The corresponding signal in the AS treatment was "I indicate that my play is [A or B]" in Catalan and was provided by Jordi Brandts.
} 
TABLE I

Payoff Calibrations

\begin{tabular}{|c|c|c|c|c|c|c|c|c|c|c|c|}
\hline & \multicolumn{8}{|c|}{ Stag Hunt } & & \multicolumn{2}{|c|}{ Prisoner's Dilemma } \\
\hline & \multicolumn{2}{|c|}{ (1) } & & \multicolumn{2}{|c|}{ (2) } & & \multicolumn{2}{|c|}{ (3) } & & \multirow[b]{2}{*}{ A } & \multirow[b]{2}{*}{$\mathrm{B}$} \\
\hline & A & $\mathrm{B}$ & & A & $\mathrm{B}$ & & A & $\mathrm{B}$ & & & \\
\hline A & 70,70 & 80,50 & A & 70,70 & 80,10 & A & 70,70 & 80,0 & A & 70,70 & 120,10 \\
\hline B & 50,80 & 90,90 & B & 10,80 & 90,90 & B & 0,80 & 90,90 & B & 10,120 & 90,90 \\
\hline
\end{tabular}

ments, the receivers made their decisions after these message forms were completed and distributed.

\section{RESULTS}

Aggregated results are presented below. Detailed outcomes for each sixperson cell in each treatment, as well as individuals' response to B signals, can be found in the working-paper version and are available upon request.

\section{Signal Followed by Action (SA)}

The results are striking. In the stag hunt variations, the proportion of B signals was $95 \%$. Conditional on a B signal, B was played by both the sender and the receiver with $94 \%$ probability and the efficient outcome achieved in 282 of 315 cases $(89.5 \%)$. Overall, the proportion of B plays was $91 \%$ and the likelihood of the efficient outcome was $86 \%$. Results are presented in the Cooper et al. (1992) format in Table II.

The results for these three calibrations are quite similar. There is no appreciable difference for either the proportion of B plays $(0.90,0.92$, and 0.91 for Games 1, 2, and 3, respectively) or the proportion of [B,B] outcomes $(0.84,0.88$, and 0.86$)$. A Kruskal-Wallis test, using each sextuple as one observation, fails to reject the hypothesis that the behavior is the same across calibrations $\left(\chi^{2}=0.268, d . f .=2\right.$, n.s. $)$.

An examination of the behavior of the receivers shows that the vast majority of subjects considered a B signal to be credible. $83 \%$ of subjects always responded to a B signal by playing B. Overall, 61 of 66 receivers $(92 \%)$ played $\mathrm{B}$ at least $75 \%$ of the time when a B signal was received. Given the senders' behavior in every sextuple, a B play was ex post payoffmaximizing for a receiver and thus it was also optimal for a sender to signal $\mathrm{B}$ and to then play $\mathrm{B}$.

There was little change over time, although $[\mathrm{B}, \mathrm{B}]$ rates were slightly lower in the first and last periods. 
TABLE II

Signal, then Action

\begin{tabular}{lccccc}
\hline \multicolumn{5}{c}{ Game (1) } & \\
Signaler, Receiver play & \\
Announcement & A,A & B,B & A,B & B,A & Total \\
\hline A & 4 & 1 & 2 & 1 & 8 \\
B & 1 & 100 & 4 & 7 & 112 \\
Total & 5 & 101 & 6 & 8 & 120
\end{tabular}

Game (2)

Signaler, Receiver play

\begin{tabular}{lccccc} 
Announcement & A,A & B,B & A,B & B,A & Total \\
\hline A & 2 & 1 & 2 & 1 & 6 \\
B & 1 & 105 & 3 & 5 & 114 \\
Total & 3 & 106 & 5 & 6 & 120
\end{tabular}

Game (3)

Signaler, Receiver play

\begin{tabular}{lccccc} 
Announcement & A,A & B,B & A,B & B,A & Total \\
\hline A & 1 & 0 & 0 & 0 & 1 \\
B & 2 & 77 & 8 & 2 & 89 \\
Total & 3 & 77 & 8 & 2 & 90
\end{tabular}

Total-Game (1), (2), (3)

Signaler, Receiver play

\begin{tabular}{lccccc} 
Announcement & A,A & B,B & A,B & B,A & Total \\
\hline A & 7 & 2 & 4 & 2 & 15 \\
B & 4 & 282 & 15 & 14 & 315 \\
Total & 11 & 284 & 19 & 16 & 330 \\
\hline
\end{tabular}

No Signals (NS)

In order to test the effect of the signal, it is necessary to compare these results with the play in the sessions without communication. (See Table III.)

There is a trend toward more risk-dominant (A) plays with a higher degree of risk-dominance. However, even if we make the rather dubious assumption that every decision is independent, only the difference between the proportions of $\mathrm{B}$ plays in games 1 and 3 are significant at conven- 
TABLE III

No Signal

\begin{tabular}{lccccc}
\hline Game & B plays & A plays & [B,B] & Off-diagonal & {$[\mathrm{A}, \mathrm{A}]$} \\
\hline$(1)$ & $40 \%$ & $60 \%$ & $21 \%$ & $39 \%$ & $40 \%$ \\
$(2)$ & $35 \%$ & $65 \%$ & $13 \%$ & $44 \%$ & $43 \%$ \\
$(3)$ & $29 \%$ & $71 \%$ & $17 \%$ & $23 \%$ & $60 \%$ \\
\hline
\end{tabular}

tional levels. ${ }^{16}$ A Kruskal-Wallis test, using each sextuple as one observation, fails to reject the hypothesis that behavior is the same in all three games $\left(\chi^{2}=0.825\right.$, d.f. $=2$, n.s. $)$. There is considerable variation across sextuples: Some groups begin with very high proportions of B moves and maintain substantial coordination, while others start with few B moves and rapidly converge to all A plays.

A very conservative nonparametric statistical test, the Wilcoxon-MannWhitney test on individual sextuples, shows that there is a strongly significant difference $(p<0.0001)$ between the SA and no communication treatments for both the proportion of $\mathrm{B}$ moves and the proportion of $[\mathrm{B}, \mathrm{B}]$ outcomes. It is obvious that the signal has a strong behavioral effect on play, even though theory correctly points out that a B signal is potentially a mixed message.

Here the proportion of B plays decreases steadily and substantially without communication (from $55-60 \%$ in the first two periods to $35-40 \%$ in the last two periods). Although 10 periods may not be enough to achieve equilibrium, the $[\mathrm{A}, \mathrm{A}]$ equilibrium is increasingly likely over time (30\% in the first two periods vs $55 \%$ in the last two periods) without communication, while the $[\mathrm{B}, \mathrm{B}]$ outcome drops from $25-35 \%$ in the early rounds to a fairly consistent rate of $10 \%$ thereafter. There are considerably more offdiagonal results in this treatment, although these are less common in later rounds. Ex post, it was payoff-maximizing to play A.

\section{Prisoner's Dilemma (PD)}

Since the proportion of B play is so high compared to some other results and a written signal was used rather than a computer message, a prisoner's dilemma game control was conducted as a control to investigate whether signalers played B simply because they felt "contractually bound" by their marked signals. (See Table IV.)

${ }^{16}$ Using the $\chi$-square test with $\mathrm{A}$ and $\mathrm{B}$ choices, we have $\chi^{2}=7.22(p<0.01)$ comparing games 1 and 3; comparing across games 1 and 2, we find $\chi^{2}=1.81$ (n.s.), comparing games 2 and $3, \chi^{2}=2.56$ (n.s.). The critical value for the $5 \%$ significance level (one degree of freedom) is 3.84 . 
TABLE IV

Prisoner's Dilemma

\begin{tabular}{lccccc}
\hline & \multicolumn{5}{c}{ Signaler, Receiver play } \\
Announcement & A,A & B,B & A,B & B,A & Total \\
\hline A & 15 & 1 & 2 & 1 & 19 \\
B & 59 & 1 & 5 & 6 & 71 \\
Total & 74 & 2 & 7 & 7 & 90 \\
\hline
\end{tabular}

Here there were only $10 \%$ B plays overall, despite the fact that the proportion of B signals was $79 \%$. The probability of a B play conditioned on a B signal was only $12 \%$ for the signaler and $10 \%$ for the receiver. There is very little change in the rate of false signals over the course of the 10 periods. Senders clearly did not feel bound to comply with their written messages and receivers did not view these signals as credible. While the urge towards payoff-dominance may be high, this influence can be overcome by clear disincentives.

The PD results show that subjects are willing to misrepresent their intentions when it suits them, even with a written signal. However, a written signal in the SA treatment may still potentially lead to different behavior than a computer signal. It may well be true that there is a greater aversion to misrepresentation with a written signal than otherwise. Any such degree of aversion must be considered, along with the underlying environment and payoff structure, when assessing the credibility of a message.

\section{Action Followed by Signal (AS)}

When it is common knowledge that senders have already chosen their actions, some subjects appear to focus more on the self-interest problem noted by Aumann. Results were highly group-specific. For the six groups, two featured nearly all-B play, two quickly became nearly all-A, and the other two were still mixed after 10 periods. Thus, in some cases it may be that an urge toward payoff-dominance is strong enough to induce and sustain B plays. In others, cooperation breaks down rapidly and completely. Aggregated results are presented in Table V.

B signals were still the norm (82\%), but the overall probability of a B play, given a B signal $(p[B \mid s(B)])$, is only 0.52 . However, the variance is quite high and the probability by sextuple ranges from a low of 0.07 (3 of 46) to a high of 1.00 (60 of 60). [A,A] rates increase steadily over time, from $30 \%$ in the beginning to over $50 \%$ at the end. [B,B] rates drop from $55 \%$ to under $40 \%$.

As before, we can use the Wilcoxon test on sextuples to test whether the results for these sextuples differ significantly from those for groups in 
TABLE V

Action, then Signal

\begin{tabular}{lccccc}
\hline & \multicolumn{5}{c}{ Signaler, Receiver play } \\
Announcement & A,A & B,B & A,B & B,A & Total \\
\hline A & 32 & 0 & 0 & 1 & 33 \\
B & 48 & 68 & 15 & 16 & 147 \\
Total & 80 & 68 & 15 & 17 & 180 \\
\hline
\end{tabular}

other treatments. Comparing the (AS) results with data from the SA treatment, we can reject the hypothesis that there is no difference in treatments at $p=0.05(z=1.96) .{ }^{17}$ On the other hand, this test cannot reject the corresponding hypothesis for the NS/AS comparison $(z=0.33$, n.s.).

However, a test which treats each sextuple as only one observation may be too conservative to generate enough power to reject a false null. At least two alternative tests might be considered. First, there is the standard test which treats each choice is an i.i.d. draw (and ignores complications of population interdependence). If our metric is either the percentage of B plays or the percentage of $[\mathrm{B}, \mathrm{B}]$ outcomes, the test of proportions (Glasnapp and Poggio, 1985) finds that the AS and NS treatments differ at $p<0.01$.

Second, a moderately conservative test examines the data from only one period and treats each person or outcome as a data point, pooling across sextuples within conditions. Here we find borderline statistical differences. If we focus on the percentage of B play, this test finds a difference significant at $p=0.19(z=1.31)$; if we test the percentage of $[\mathrm{B}, \mathrm{B}]$ outcomes, the difference is significant at $p=0.06(z=1.87) .{ }^{18}$ Using this test for the AS/SA comparison gives significance at $p<0.001$, using either metric ( $z=4.14$ or $z=3.38$, respectively).

Thus, while even the most conservative test rejects $\mathrm{SA}=\mathrm{AS}$, this same test cannot reject $\mathrm{AS}=\mathrm{NS}$. However, other tests suggest there may be a small difference between these two treatments: Comparing the AS and NS results, even a signal which follows an action choice increases the likelihood of a $[\mathrm{B}, \mathrm{B}]$ outcome; the overall rate of B play differs less. The likelihood that such a difference is due to chance cannot be judged reliably without a precise model of the nature of dependence of subjects across periods.

${ }^{17}$ The data from the three SA calibrations is pooled, given that the Kruskal-Wallis test finds little difference across the games. Similarly, the data from the NS treatment is pooled for the Wilcoxon test against the AS treatment. The significance levels reported are for the two-tailed test of differences.

${ }^{18}$ The one-period test presumably reduces the dependence within sextuples. However, it is unclear which period should be used for the test. Subjects may be confused in the 1st period; unusual behavior is not uncommon in the last period of a session. The tests are performed using third-period data, which happen to be quite representative of the overall data. 
TABLE VI

Clark et al. Game Matrix

\begin{tabular}{lccccc}
\hline & \multicolumn{5}{c}{ Signaler, Receiver play } \\
Announcement & A,A & B,B & A,B & B,A & Total \\
\hline A & 0 & 0 & 0 & 0 & 0 \\
B & 4 & 101 & 9 & 6 & 120 \\
Total & 4 & 101 & 9 & 6 & 120 \\
\hline
\end{tabular}

\section{Clark et al. and Cooper et al. Replications}

Given that my results differ in degree from those of Clark et al. and Cooper et al., it seems worthwhile to explore the cause of this difference. ${ }^{19}$ I conducted two follow-up sessions for each of these games, using one-way communication (SA) and nominal payoff matrices $(10$ points $=1$ peseta $)$ identical to the pertinent games. (See Tables VI and VII.)

The Clark et al. calibration results are very similar to those in my SA games. The Clark et al. experiment used two-way communication; this may partially explain the difference between their results and mine. In any event, behavior in the Barcelona subject pool is consistent across these games.

The results for the Cooper et al. game show somewhat less coordination on $[\mathrm{B}, \mathrm{B}]$, only $72 \%$ of all outcomes. This compares to the $65 \%$ observed for one-way communication in the first 11 rounds of the Cooper et al. experiment. ${ }^{20}$ However, this is due almost entirely to the greater likelihood of an A signal ${ }^{21}$ - a B signal leads to $86 \%$ [B,B] outcomes, just lower than the $89.5 \%$ in my SA treatment. In fact, the probability of a B play given a $\mathrm{B}$ signal is quite consistent across the various payoff matrices-

${ }^{19}$ I thank two anonymous reviewers for this suggestion.

${ }^{20}$ The 1992 paper reports only the data from the last 11 periods of their 22 period session. My thanks to Doug DeJong for providing the complete data set.

${ }^{21}$ With this payoff matrix, the sender does not strictly prefer that the receiver plays $\mathrm{B}$, so it should not be surprising that A signals are more frequent. The preponderance $(20 / 23$, or $87 \%$ ) of senders who play A also signal A.

TABLE VII

Cooper et al. Game Matrix

\begin{tabular}{lccccc}
\hline & \multicolumn{5}{c}{ Signaler, Receiver play } \\
Announcement & A,A & B,B & A,B & B,A & Total \\
\hline A & 19 & 0 & 1 & 0 & 20 \\
B & 0 & 86 & 3 & 11 & 100 \\
Total & 9 & 86 & 4 & 11 & 120 \\
\hline
\end{tabular}


TABLE VIII

\begin{tabular}{lcccccccc}
\hline & $\begin{array}{c}\mathrm{B} \\
\text { Treatment }\end{array}$ & $\begin{array}{c}\mathrm{A} \\
\text { plays }\end{array}$ & {$[\mathrm{B}, \mathrm{B}]$} & {$[\mathrm{A}, \mathrm{A}]$} & $\begin{array}{c}\text { Off- } \\
\text { diagonal }\end{array}$ & $\mathrm{p}[\mathrm{s}(\mathrm{B})]$ & $\begin{array}{c}\mathrm{p}[\mathrm{B} \mid \mathrm{s}(\mathrm{B})] \\
\text { (Sender) }\end{array}$ & $\begin{array}{c}\mathrm{p}[\mathrm{B} \mid \mathrm{s}(\mathrm{B})] \\
(\text { Receiver })\end{array}$ \\
\hline SA & $91 \%$ & $9 \%$ & $86 \%$ & $11 \%$ & $3 \%$ & 0.955 & 0.940 & 0.943 \\
NS & $35 \%$ & $65 \%$ & $16 \%$ & $47 \%$ & $37 \%$ & & & \\
AS & $47 \%$ & $53 \%$ & $38 \%$ & $44 \%$ & $18 \%$ & 0.817 & 0.571 & 0.565 \\
PD & $10 \%$ & $90 \%$ & $2 \%$ & $82 \%$ & $16 \%$ & 0.789 & 0.099 & 0.085 \\
Clark & $90 \%$ & $10 \%$ & $84 \%$ & $3 \%$ & $13 \%$ & 1.000 & 0.892 & 0.917 \\
Cooper & $78 \%$ & $22 \%$ & $72 \%$ & $16 \%$ & $12 \%$ & 0.833 & 0.970 & 0.890 \\
\hline
\end{tabular}

94\% in my SA games, $91 \%$ with the Clark et al. payoff matrix, and $93 \%$ with the Cooper et al. payoff matrix).

A comparison across all treatments is shown in Table VIII.

\section{DISCUSSION}

The degree of coordination with a one-way signal is quite high when the signal precedes an action. Coordination and efficiency are much lower without a signal, so that the hypothesis that the signal conveys no information is easily rejected. The results in the prisoner's dilemma game show that it is possible for a game's payoff structure to overcome tendencies to adhere to one's signal, so that a written signal cannot per se always be taken at face value.

In addition, reversing the order of the sender's decisions can induce a major difference in behavior. In the AS treatment, some groups can coordinate on $[\mathrm{B}, \mathrm{B}]$ outcomes, while others coordinate on $[\mathrm{A}, \mathrm{A}]$ outcomes. While the AS results do differ significantly from the SA results, they cannot be statistically distinguished from those obtained without communication. In this sense, Farrell's comment on Aumann's conjecture is borne out by the data. Perhaps the reversal of the order of signal and action brings the cognitive task and self-interest issue into sharper focus, reducing the credibility of a signal. While the SA sequence may seem the more natural interpretation, the AS sequence is potentially more realistic in some economic environments.

Payoff-dominance seems to be a strong influence here, although riskdominance usually trumps it when no communication is possible or when the credibility of a signal is perceived to be low. The prisoner's dilemma control shows that risk-dominance is pre-eminent when there are strong disincentives for attempting to reach efficiency, regardless of whether the efficient move has been signaled. 
TABLE IX

Payoff Efficiency

\begin{tabular}{lccccc}
\hline Treatment & $\begin{array}{c}\text { \# of } \\
\text { subjects }\end{array}$ & $\begin{array}{c}\text { Average } \\
\text { payoff }\end{array}$ & $\begin{array}{c}\text { Expected } \\
\text { random payoffs }\end{array}$ & $\begin{array}{c}\text { Maximum } \\
\text { possible payoffs }\end{array}$ & Efficiency \\
\hline SA1 & 24 & 86.25 & 72.5 & 90 & 0.786 \\
SA2 & 24 & 85.37 & 62.5 & 90 & 0.832 \\
SA3 & 18 & 83.78 & 60 & 90 & 0.793 \\
Avg. SA & 66 & & & & $\mathbf{0 . 8 0 4}$ \\
NS1 & 24 & 72.21 & 72.5 & 90 & -0.017 \\
NS2 & 36 & 59.22 & 62.5 & 90 & -0.119 \\
NS3 & 24 & 66.75 & 60 & 90 & 0.225 \\
Avg. NS & 84 & & & & $\mathbf{0 . 0 3 0}$ \\
AS & 36 & 72.22 & 62.5 & 90 & $\mathbf{0 . 3 5 4}$ \\
Clark & 24 & 92.12 & 65 & 100 & $\mathbf{0 . 7 7 5}$ \\
Cooper & 24 & 89.33 & 65 & 100 & $\mathbf{0 . 6 9 5}$ \\
\hline
\end{tabular}

It is not merely a taste for honesty or a perception of an implicit contract that drives the high degree of coordination we observe here in the SA games. Subjects may anticipate that other players would believe a B signal, making a B play the best choice. Yet there seems to be more uncertainty about credibility when the order is reversed-perhaps this sequence is seen to highlight the self-serving nature of the signal. For sophisticated groups of players, behavior in SA and AS treatments might not differ.

We can attempt to assess the economic significance of a signal's effectiveness by creating a payoff efficiency index. Without a signal, each payoff calibration would yield some expected per-player payoff if decisions were made randomly and with equal probability. In the stag hunt, there is also a clearly defined Pareto-optimal set of payoffs. Consider an efficiency index, $e=\left(\Pi_{a}-\Pi_{r}\right) /\left(\Pi_{m}-\Pi_{r}\right)$, where the subscripts refer to actual average payoffs, maximum possible payoffs, and expected random-action payoffs, respectively. (See Table IX.)

Communication induces an $80 \%$ efficiency level in the SA treatment, with an individual obtaining, on average, about $95 \%$ of the maximum possible payoff of 90. Efficiency is just slightly lower for the Clark et al. and Cooper et al. calibrations. The average payoff achieved in the no signal condition is quite close to that expected with random actions. ${ }^{22}$ Even the AS efficiency level is substantially better than zero. AS average payoffs are higher when a B signal is made (73.98 to 69.24 for an A signal), but any signal does

\footnotetext{
${ }^{22}$ The resulting near-zero efficiency level makes the normalization used at least potentially plausible.
} 
better than the random-action expected payoff of 62.5. ${ }^{23}$ Although in this case a B signal is often not considered credible, AS signals are at least fairly successful in achieving coordination on one of the two on-diagonal payoff cells.

While results differ somewhat from those of previous studies, the message technology, subject pool, and other factors differ. In the first 11 periods of the original Cooper et al. game, there was a $65 \%$ chance of a [B,B] outcome and a probability of 0.82 that a receiver played $\mathrm{B}$ in response to a one-way B signal. Without signals, these periods show a $10 \%$ probability for the $[\mathrm{B}, \mathrm{B}]$ outcome and a $55 \%$ chance for the $[\mathrm{A}, \mathrm{A}]$ outcome, with the proportion of $\mathrm{B}$ moves at 0.28 .

The replication with the Clark et al. payoff matrix gives results quite similar to those in my SA treatments. Many potential explanations can be proposed for the difference in results across our sessions. In an Aumann game, inferences with two-way simultaneous signals may be more complex and the credibility of a B signal thus diminished. Second, a written signal may be more credible than a computer signal, although the prisoner's dilemma treatment shows that this written form per se is insufficient to achieve Pareto-efficiency. Finally, with heterogeneity across subject pools, a plausible difference in subjects' beliefs about the beliefs of others could explain much of the contrast in behavior. ${ }^{24}$ No definitive conclusion about the difference in results can be drawn at this time; however, in all cases pre-play communication with the SA decision order strongly enhances the degree of coordination.

\section{CONCLUSION}

Achieving coordination in economic contexts is an important issue. Aumann (1990) conjectures a limitation on the effectiveness of pre-play communication as a coordination mechanism. Yet when his example is tested with a signal followed by an action, the conjecture is rejected. However, as

\footnotetext{
${ }^{23}$ Curiously, the likelihood of an $[\mathrm{A}, \mathrm{A}]$ outcome given a $\mathrm{B}$ signal is actually slightly higher than the probability of an $[\mathrm{A}, \mathrm{A}]$ outcome without a signal. Table 4 shows that $61 \%(48 / 79)$ of B signals led to [A,A] outcomes, compared to the 55\% (197/360) probability of an [A,A] outcome in the NS treatment. This result may be analogous to the nonconsequentalist reasoning discussed by Shafir and Tversky (1992) and Croson (1999), where (in a prisoner's dilemma) a player is more likely to cooperate when he does not know the action of the other player than when he knows the other player has chosen the cooperative move.

${ }^{24}$ Note that B signals were made only $81 \%$ of the time in their Aumann's conjecture test; moreover, in a third Clark et al. game where players have a clear incentive to report truthfully, the chance of a B signal is only $51.5 \%$ and the likelihood of B play is only about $29.5 \%$ (this figure provided by Martin Sefton).
} 
Farrell suggests, the results are rather different when the order of decisions is known to be reversed. From a purely theoretical perspective, there is no obvious reason why the decision order should matter. Nonetheless, the transparency of the cognitive task appears to critically affect the credibility of the signal.

One cannot assume that the observed laboratory behavior is completely general. However, in a natural environment there may be other factors that enhance coordination-for example, there is often a serious issue of reputation. To the extent to which the laboratory abstracts actual behavior, the results have direct implications for coordination possibilities.

There are a number of extensions for this research; for example, there may be different "degrees" of cheap talk. ${ }^{25}$ Radner and Schotter (1989) and Schotter et al. (1992) find that bargaining is more efficient with more personal communication. In this case, it may be perceived as more of a breach of a social norm to violate a technically non-binding agreement. Situations where payoffs are more in conflict could require higher cheap talk intensities. Dawes et al. (1977) find that personal communication produces significantly more public good provision in a dilemma situation, so that cheap talk may even be beneficial in achieving cooperation in more difficult environments. Weber et al. (1999) use cheap talk in a minimum-effort (multi-person stag hunt) game to investigate the effects of "leadership" on the likelihood of Pareto-optimal choices, operationalizing the concept of degree as the fraction of people who speak at once-the higher the fraction, the more coordination.

Much of the interaction in the world resembles cheap talk, in that implicit agreements are only indirectly enforceable. This study shows that one-way communication can be quite effective in achieving coordination, even when there are valid theoretical reasons for doubting the content of a message. Payoff-dominance is a strong element of the equilibrium-selection process in this experiment, although risk-dominance generally prevails if no signal can be made and can also prevail when actions precede signals.

\section{REFERENCES}

Aumann, R. (1990). "Nash-Equilibria are not Self-Enforcing," in Economic Decision Making: Games, Econometrics and Optimisation (J. Gabszewicz, J.-F. Richard, and L. Wolsey, Eds.), pp. 201-206. Amsterdam: North-Holland.

Blume, A. (1998). "Communication, Risk, and Efficiency in Games," Games Econom. Behavior 22, 171-202.

\footnotetext{
${ }^{25} \mathrm{An}$ increasing ranking might be disembodied computer signals, written messages, visual identification, verbal contact, and face-to-face communication.
} 
Cachon, G., and Camerer, C. (1996). "Loss-Avoidance and Forward Induction in Experimental Coordination Games,” Quart. J. of Econ. 111, 165-194.

Clark, K., Kay, S., and Sefton, M. (1997). "When are Nash Equilibria Self-Enforcing? An Experimental Analysis," mimeo.

Cooper, R., DeJong, D., Forsythe, R., and Ross, T. (1990). "Selection Criteria in Coordination Games: Some Experimental Results," Amer. Econ. Rev. 80, 218-233.

Cooper, R., DeJong, D., Forsythe, R., and Ross, T. (1992). "Communication in Coordination Games," Quar. J. of Econ. 53, 739-771.

Croson, R. (1999). "The Disjunction Effect and Nonconsequential Reasoning in Dominant Strategy Games: Extensions and Limitations," Organizational Behavior and Decision Process, in press.

Dawes, R., McTavish, J., and Shaklee H. (1977). "Behavior, Communication, and Assumptions about Other People's Behavior in a Commons Dilemma Situation," J. Personality and Social Psychology 35, 1-11.

Farrell, J., and Rabin, M. (1996). "Cheap Talk,” J. Econom. Perspectives 10, 103-118.

Farrell, J. (1988). "Communication, Coordination, and Nash Equilibrium,” Econom. Lett. 27, 209-214

Glasnapp, D., and Poggio, J. (1985). Essentials of Statistical Analysis for the Behavioral Sciences, Columbus, OH: Merrill.

Harsanyi, J. (1995). "A New Theory of Equilibrium Selection for Games with Complete Information," Games Econom. Behavior 8, 91-122.

Harsanyi, J., and Selten, R. (1988). A General Theory of Equilibrium Selection in Games, Cambridge, MA: MIT Press.

Hurkens, S. (1996). "Multi-sided Pre-play Communication by Burning Money,” J. Econ. Theory 69, 186-97.

Isaac, M., and Walker, J. (1988). "Group Size Effects in Public Goods Provision: The Voluntary Contributions Mechanism," Quart. J. Econom. 103, 179-199.

Ochs, J. (1995). "Coordination Problems," in The Handbook of Experimental Economics (J. Kagel and A. Roth, Eds.), pp. 195-251. Princeton: Princeton University Press.

Radner, R., and Schotter, A. (1989). "The Sealed-Bid Mechanism: An Experimental Study," J. Econom. Theory 48, 179-220.

Schotter, A., Snyder, B., and Zheng, W. (1992). "Bargaining through Agents: An Experimental Study," mimeo.

Shafir, E., and Tversky, A. (1992). "Thinking through Uncertainty: Nonconsequential Reasoning and Choice," Cognitive Psychology 24, 449-474.

Van Huyck, J., Battalio, R., and Beil, R. (1990). "Tacit Coordination Games, Strategic Uncertainty and Coordination Failure," Amer. Econ. Rev. 80, 234-248

Van Huyck, J., Battalio, R., and Beil, R. (1993). "Asset Markets as an Equilibrium Selection Mechanism: Coordination Failure, Game Form Auctions, and Tacit Communication," Games Econom. Behavior 5, 485-504.

Weber, R., Rottenstreich, Y., Camerer, C., and Knez, M. (1999). "The Illusion of Leadership: Misattribution of Cause in Coordination Games," mimeo. 